\title{
SPES: EXOTIC BEAMS FOR NUCLEAR PHYSICS STUDIES
}

\author{
ALBERTO ANDRIGHETTO, MATTIA MANZOLARO, STEFANO CORRADETTI, DANIELE SCARPA, \\ JESU VASQUEZ, MASSIMO ROSSIGNOLI, ALBERTO MONETTI, \\ MICHELE CALDEROLLA, GIANFRANCO PRETE
}

INFN Laboratori Nazionali di Legnaro Via dell'Università 2, 35020 Legnaro (Padova), Italy andrighetto@Inl.infn.it

Published 25 February 2014

\begin{abstract}
The SPES project at Laboratori di Legnaro of INFN (Italy) is concentrating on the production of neutron-rich radioactive nuclei for nuclear physics experiments using uranium fission at a rate of $10^{13} \mathrm{fission} / \mathrm{s}$. The emphasis on neutron-rich isotopes is justified by the fact that this vast territory has been little explored. The Radioactive Ion Beam (RIB) will be produced by the ISOL technique using proton induced fission on a direct target of UCx. The most critical element of the SPES project is the Multi-Foil Direct Target. Up to the present time, the proposed target represents an innovation in terms of its capability to sustain the primary beam power. This talk will present the status of the project financed by INFN, which is actually in the construction phase at Legnaro. In particular, developments related to the target and the ion-source activities using the surface ion source, plasma ion source, and laser ion source techniques will be reported.
\end{abstract}

Keywords: ISOL facility; cyclotron; fission; target; ion source.

\section{Introduction}

SPES ${ }^{1}$ is the acronym for "Selective Production of Exotic Species". The aim of this ISOL (Isotope Separation On-Line) facility ${ }^{2}$ is to deliver high intensity and high-quality beams of neutron-rich nuclei to perform forefront research in nuclear structure, reaction dynamics and interdisciplinary fields like medical, biological and material sciences. SPES represents an intermediate step toward the future generation European ISOL facility EURISOL. ${ }^{3}$ The SPES project is supported by the Italian national laboratories LNL (Legnaro) and LNS (Catania). It is based on the ISOL method with an UCx Direct Target able to sustain a power of $10 \mathrm{~kW}$.

The primary proton beam is delivered by a cyclotron accelerator with energy up to 70 $\mathrm{MeV}$ and a beam current of $0.75 \mathrm{~mA}$. Neutron-rich radioactive ions will be produced by uranium fission target ${ }^{4}$ at an expected fission rate in the target of the order of $10^{13}$ fissions

This is an Open Access article published by World Scientific Publishing Company. It is distributed under the terms of the Creative Commons Attribution 3.0 (CC-BY) License. Further distribution of this work is permitted, provided the original work is properly cited. 
per second. The exotic isotopes will be re-accelerated by the ALPI superconducting LINAC $^{5}$ at energies of $10 \mathrm{AMeV}$ and higher for masses in the region of $\mathrm{A}=130 \mathrm{amu}$, with an expected rate on the secondary target up to $10^{9}$ particles per second.

\section{Investigations on Nuclear Physics at SPES}

Starting from a nucleus on the stability line and adding successive neutrons, one observes that the binding energy of the last neutron decreases steadily until it vanishes and the nucleus decays by neutron emission. The position in the nuclear chart where this happens defines the neutron drip line. It lies much farther away from the valley of stability than the corresponding drip line associated with protons, owing to the absence of electrical repulsion between neutrons.

The location of the neutron drip line is largely unknown as experimental data are available only for nuclei with mass up to around 30. The interest in the study of nuclei with large neutron excess is not only focused on the location of the drip line but also on the investigation of the density dependence of the effective interaction between the nucleons for exotic N/Z ratios.

The evolution of nuclear properties towards the neutron drip line depends on how the shell structure changes as a function of neutron excess. This evolution has consequences on the ground state properties of the nuclei and on the single-particle and collective excitations. In particular, studies of neutron-rich nuclei beyond the doubly magic ${ }^{132} \mathrm{Sn}$ are of key importance to investigate the single-particle structure above the $\mathrm{N}=82$ shell closure and find out how the effective interaction between valence nucleons behaves far from stability.

New modes of collective motion are also expected in connection with the formation of a neutron skin, namely oscillation of the skin against the core, similar to the soft dipole mode already identified in the case of very light halo nuclei. Presently, neither the thickness nor the detailed properties of the neutron skin of exotic nuclei are known.

This information is needed to enable a quantitative description of compact systems like neutron stars, where exotic nuclei forming a Coulomb lattice are immersed in a sea of free neutrons, a system which is expected to display the properties of both finite and infinite (nuclear matter) objects. At the energy of SPES, it will be possible to address important questions related to the study of neutron-rich matter such as nuclear forces, level density, viscosity, barrier, neutron pairing and collective modes.

\section{The SPES ISOL Facility}

The radioactive ions will be produced with the ISOL technique using the proton induced fission on a Direct Target of $\mathrm{UCx}^{6}$ and subsequently reaccelerated using the PIAVEALPI accelerator complex. ${ }^{5}$ A uranium fission rate of $10^{13}$ fission/s is foreseen. A cyclotron with a maximum current of $0.8 \mathrm{~mA}$ and two exit ports will be used as a proton driver accelerator with variable energy $(30-70 \mathrm{MeV}){ }^{7}$ The BEST cyclotron is a compact four straight-sector machine, energized by a pair of room temperature conducting coils. 
The cyclotron is able to accelerate $\mathrm{H}^{-}$beams provided by an external multi-cusp ion source up to the energy of $70 \mathrm{MeV}$. Since the proton extraction is done by the stripping process, the final energy varies from $35-70 \mathrm{MeV}$. Two independent extraction channels placed at $180^{\circ}$ with respect to each other provide the simultaneous delivery of two beams. The maximum beam current deliverable is estimated to be $800 \mu \mathrm{A}$. The cyclotron will be equipped with two extraction combination magnets placed at $180^{\circ}$ with respect to each other. Any proton beam extracted by the $\mathrm{H}^{-}$stripping process in the energy range of 35-70 MeV will be transported at a crossover point inside one of the extraction magnets (Fig. 1). Figure 1 shows a picture of the SPES cyclotron, which is actually in the construction phase at the Best Theratronics factory.

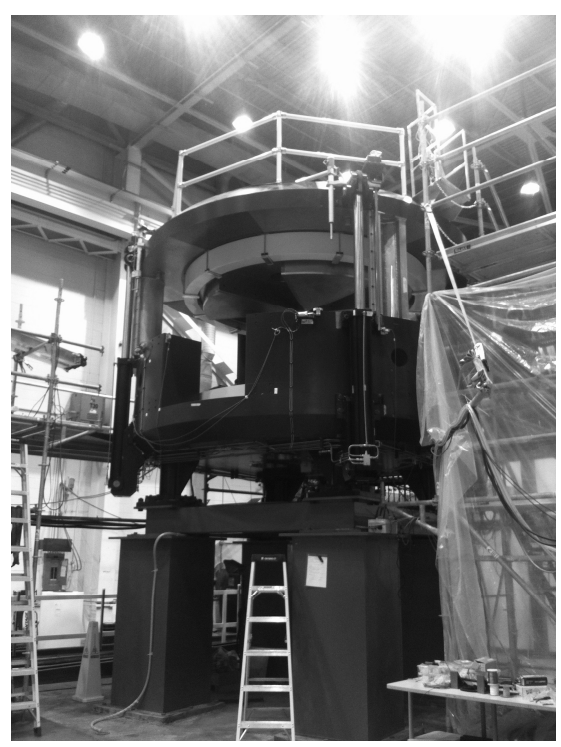

Fig. 1. The cyclotron, the proton driver of SPES.

In order to reach a fission rate of $10^{13}$ fission/s, a proton beam current of $200 \mu \mathrm{A}$ (at $40 \mathrm{MeV}$ ) is necessary. The secondary beam, up to $500 \mu \mathrm{A}$ at $70 \mathrm{MeV}$, will be devoted to applications, mainly neutron production for material research and study of new isotopes for medical applications.

The ISOL technique for radioactive beam production is based on a driver accelerator that induces nuclear reactions inside a thick target. The reaction products are extracted from the target by a thermal process, ionized and injected into a re-accelerator. The linear accelerator ALPI, with $\mathrm{CW}$ operation and $\beta(v / c)$ ranging from $\sim 0.04$ to 0.2 , represents an ideal re-accelerator for radioactive beams. Radioactive ions can be accelerated above the Coulomb barrier with high efficiency and with a quasi-continuous time structure well suited for experiments. A time structure suitable for TOF measurements can be implemented by a low energy bunching system. In recent years ALPI underwent a number of significant upgrades, which made it a world-class facility in heavy ion stable 


\section{A. Andrighetto et al.}

beam accelerators and, in turn, an important added value for its use as an RIB accelerator as well. ALPI has an equivalent acceleration voltage of $40 \mathrm{MV}$ and can accelerate heavy ions in the region of tin at energies between 6 and $13 \mathrm{AMeV}$, according to their charge state (19+ or 40+ respectively). Figure 2 is a schematic layout of the SPES main building, which is in the construction phase now at LNL.

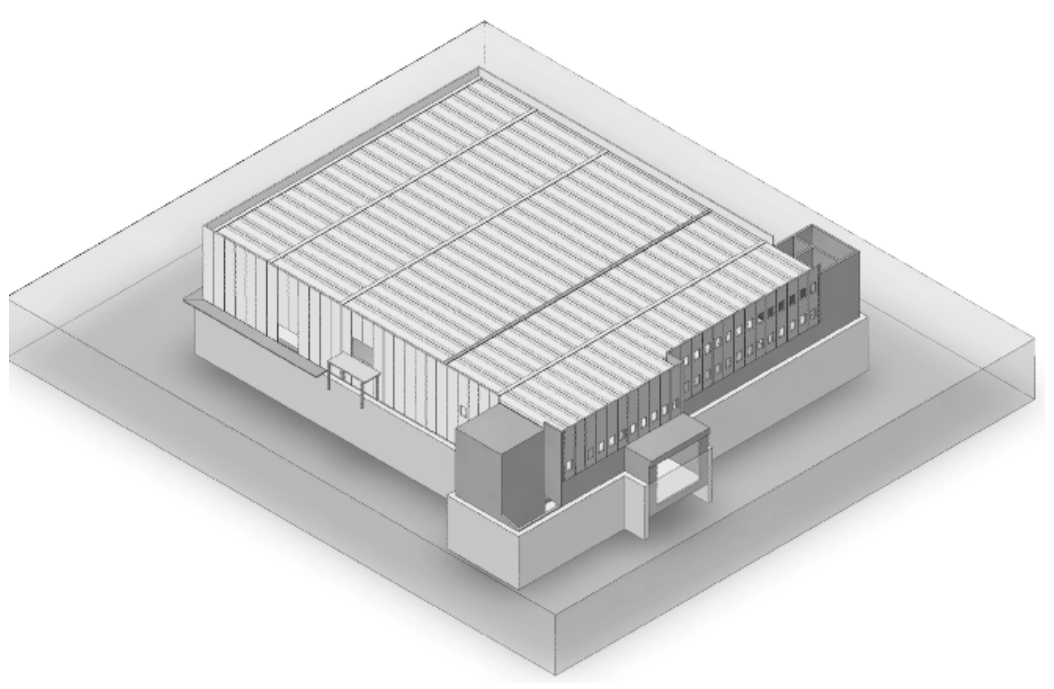

Fig. 2. The layout of the SPES building.

\section{Production Target Complex}

The most critical element of the SPES facility is the target ion source unit (TIS) where the RIB is produced. The target is composed of seven UCx disks, appropriately spaced in the axial direction, in order to dissipate the average power of $8 \mathrm{~kW}$ due to the proton beam passing through them.

The yield of nuclear isotope is a function of various parameters such as sticking time, half-life, cross section, proton flux, and diffusion and effusion times. The maximum possible rate is attenuated by the efficiencies $\varepsilon$ of product diffusion, effusion, ionization and transport, respectively.

The most critical efficiency factors can be associated with the release of the exotic atoms from the target to the ion source and it can be viewed as two different processes:

- The diffusion of the isotope from the crystal lattice to the grain boundary and to the surface.

The effusion from place to place until the atom reaches the ion source.

In order to design the best target, it is necessary to keep in mind, in addition to cross section and diffusion coefficient, such properties as limiting temperature, emissivity and thermal conductivity, porosity and permeability. Fast isotope diffusion and effusion are 
necessary in order to prevent their condensation on the cold region and their decay before isotopes can reach the ion source. For the above mentioned reasons, it is clear that the target composition and morphology play a key role in isotope release efficiency.

Porous UCx samples were prepared by means of thermal treatment under vacuum of $\mathrm{UO}_{2}$. Samples prepared from $\mathrm{UO}_{2}$ and graphite mixtures were labeled as $\mathrm{UO}_{2} \mathrm{C}$. The sintering process, which takes place at higher temperatures than the carbothermic reduction, can cause pores of small dimension to collapse if sintering is not appropriately controlled.

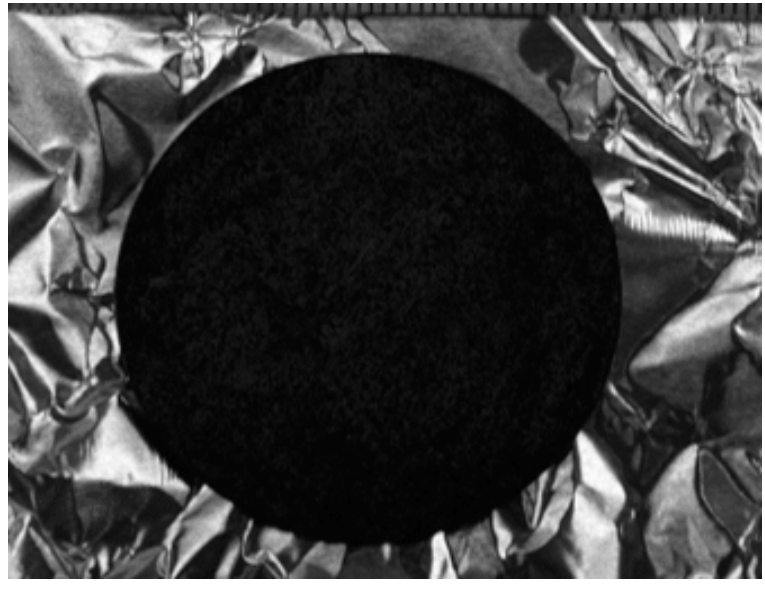

Fig. 3. The UCx target disc.

The UCx discs, shown in Fig. 3, are $40 \mathrm{~mm}$ in diameter and $1 \mathrm{~mm}$ thick. They are housed in a graphite box containing the UCx, with windows and dumps both made of graphite. The box is contained in a tantalum cylinder, which is resistively heated by passing a high current through it. ${ }^{8}$ A tantalum transfer tube connects the target unit to the ion source system into which several kinds of ion source devices can be plugged. ${ }^{9}$

In an ISOL facility such as SPES, the volatile atoms produced are released from the target material and diffuse via the transfer line into the ion source. The TIS system consists of a self-contained unit optimized for a specific element or group of elements.

The design of an ion source takes into consideration its efficiency as well as its capability of selective ionization. The appropriate choice for the TIS is of paramount importance since its performance determines the intensity, the beam quality, and the number of radioactive beams that can be provided for experimental use.

The Surface Ion Source (SIS) is the first SPES ion source developed at LNL. It is a powerful and selective device able to produce +1 ions for quite a large group of elements. In particular, the alkaline and the rare earth elements (such as $\mathrm{Rb}, \mathrm{Cs}, \mathrm{Sr}$, and $\mathrm{Ba}$ ) have a small +1 ionization potential (less than $6 \mathrm{eV}$ ), and they may be well ionized using this method, reaching high values of efficiency (up to 60\%). Some other elements like aluminum can also be ionized with this ion source but with very low efficiency $(<1 \%)$. 


\section{A. Andrighetto et al.}

The SPES front-end, where the TIS chamber is located, is an evolution of the ISOLDE FE6 and is divided into three functional subsystems. The first one is the ion source complex where the ion beam is produced. It consists of the oven device, loaded with the material to be ionized, the transfer line, the ionizer and the extractor devices. The second one is the beam optics subsystem, where the direction and focalization properties of the beam are manipulated using four electrostatic steerers and one electrostatic quadrupole triplet. The third subsystem is the beam diagnostic used to measure the relevant beam information like beam shape and intensity. This subsystem consists of a beam profile monitor, a faraday cup and the emittance meter placed at the end of the apparatus. The aluminum TIS chamber unit has a water-cooled base plate and is covered by another water-cooled bell jar. This chamber unit, which weighs about $40 \mathrm{~kg}$, is coupled to the front end by means of quick connectors.

Two valves isolate the system from the proton beam pipe and from the front end. A picture of the target-ion source complex is shown in Fig. 4. Since the target unit could be used several times, it will be shipped in a dedicated box with lead walls of $2 \mathrm{~cm}$ thickness.

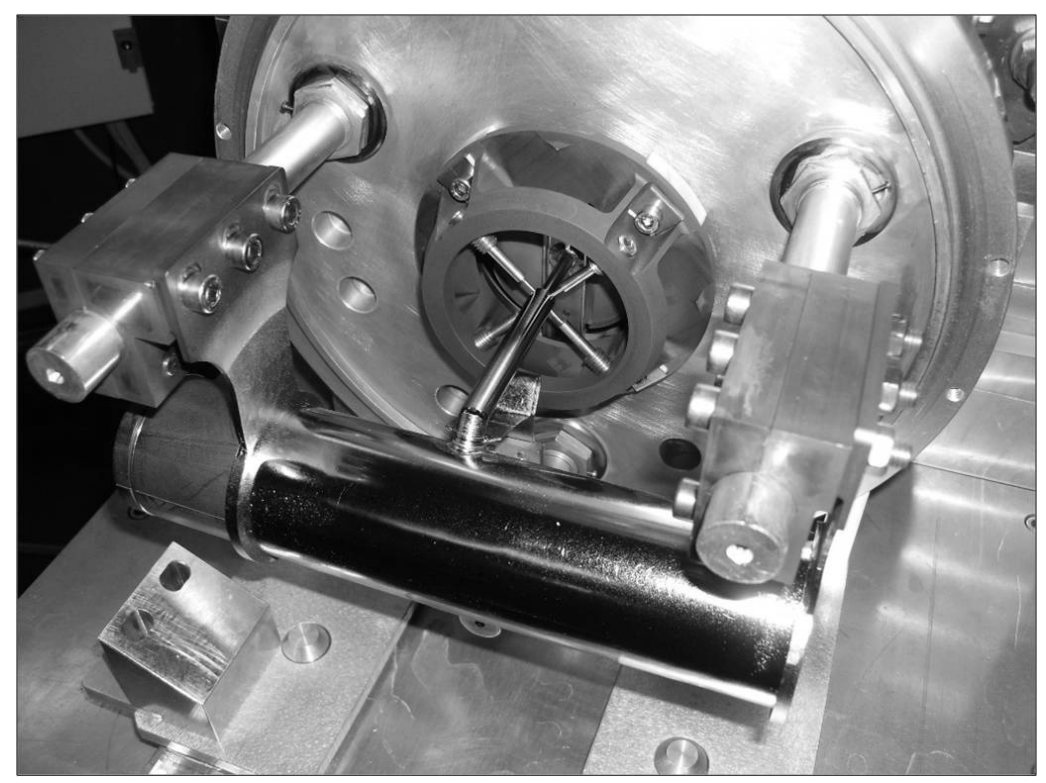

Fig. 4. The Target Ion Source (TIS) complex.

The handling system must be designed to move the target chamber unit, housed in the lead container, into the area where the target will be irradiated. Then the system should open the container, pull off the target chamber and place it onto the FE. After the irradiation stage, the target chamber must first be removed from the FE, then stored in the lead container and finally sent out of the "hot" area for further handling and long term storage. 
The lead box containing the target chamber will be housed in a special repository along with other exhausted boxes waiting for the radiation levels to reach acceptable values, allowing for subsequent phases of disposal and possible recycling. The time required for the target materials to become safe enough for inspection is around two years. After this time period, it is possible to send the target chamber unit to a dedicated laboratory (hot cell) where it can be opened for internal study in order to determine which parts can be recycled and which parts are not reusable.

\section{Conclusion}

The SPES project is one of the main nuclear physics developments in Italy for the next years. It is organized as a wide collaboration among the INFN Divisions, Italian Universities and international Laboratories. SPES is an up to date project in this field with a very competitive throughput representing a step forward for the European project EURISOL.

The relevance of the project is not only related to nuclear physics research but also to astrophysics and applied physics. The ability to simultaneously operate the ALPI Superconductive Linac and the two-exit-port Cyclotron will result in a large improvement in the research capabilities at LNL. The first exotic beam at SPES is expected in 2018.

\section{Acknowledgments}

The authors wish to thank M. Lollo, L. Costa and M. Pavan from LNL-INFN for their valuable technical support.

\section{References}

1. A. Andrighetto, G. Bisoffi et al., SPES: The INFN radioactive beam facility for nuclear physics, in AIP Conf. Proc. 1491, 58 (2012), http://dx.doi.org/10.1063/1.4764202.

2. A. C. Mueller, An Overview of Radioactive Beam Facilities, in EPAC 2000 Proc., CD-ROM, eds. J.-L. Laclare, W. Mitaroff, Ch. Petit-Jean-Genaz, J. Poole and M. Regler (Austrian Academy of Sciences Press, Vienna, 2002), p. 73.

3. J. Vevvier, Nucl. Instr. Meth. B 204, 759 (2003).

4. A. Andrighetto, C. M. Antonucci et al., Eur. Phys. Journal S.T. 150, 273 (2007).

5. A. Andrighetto, L. Biasetto, M. Manzolaro et al., Nuclear Physics A 834, 754 (2010).

6. A. Andrighetto, L. Biasetto, M. Manzolaro et al., The SPES Project at LNL, in AIP Conf. Proc. 1099, 728 (2009), http://dx.doi.org/10.1063/1.3120141.

7. M. Manzolaro, M. Manente, D. Curreli, J. Vasquez, J. Montano, A. Andrighetto et al., Rev. Sci. Instr. 83, 02A907 (2012).

8. D. Scarpa, L. Biasetto, S. Corradetti, M. Manzolaro and A. Andrighetto et al., Eur. Phys. J. A 47, 32 (2011).

9. M. Manzolaro, G. Meneghetti and A. Andrighetto, Nucl. Instr. Meth. A 623, 1061 (2010). 